Monatsschrift f. Geburtshülfe u. Gynäkologie 1930;85:I-II

\title{
Albert Döderlein
}

feiert am 5. Juli seinen JO. Geburtstag. Das L·edeutet nidit $\pi$ ur für die Familie im engeren Sinne einen hoL·en Festtag, für seine Klinik und die Stadt seiner weitreidienden Wirk-samkeit; die gesamte deutsdie Gynäkologie nimmt daran intimen Anteil und mit ihr zuversiditlich die frauenärztlidien Kreise in aller $\Lambda$ Velt. Albert Döderlein gilt L·eute als der Vertreter unseres Wissensdiaftshereidis. Er verkörpert uns die Entwidvlungsphase der Frauenkunde, weldie sidiaufL $\cdot$ aut auf die L $\cdot$ iologisdi-diemisdie und pbysikalisdie Forsdiung.

Aus seinen ersten ArL·eiten üL·er die Flora der Vagina ist in gegeL·ener Fortführung der allseitigen Forsdierarheit die heute gültige Auffassung üL·er die Phv siologie und Pathologic der weiL·lichen Genitalien hervorgegangen. Das Stidi-wort für die letztere ist die AusL·ildung des verstärkten Wundsdiutzes, die uns zum wesentlidienTeilzurGrundlage unserer 1 herapie geworden ist. Döderlein hat als erster die Bedeutung der Strahlenforsdiung audi für die Gynäkologie erkannt. indem er über die ersten so L·estedienden Er-folge $\lambda v^{\wedge}$ eitersehend auí die physikalische Erkenntnis dieser wunderL - aren Urkraft hindrängte, hat er der $\backslash$ erwendbar-keit derseíben für unsere 1 herapie die Wege gewiesen.

Als dem Lehrer und Forscher und Arzt huldigen wir ihm heute; als dem gütigen Berater und Freund, als unserm I ührer.

Die Monatssdirift für Gehurtshilfe und Gynäkologie hat sidi von ihrem AnL·eginn an seiner Anteilnahme erfieut. Mit Stolz nennen wir ihn seit 1Ç Jahren als unseren MitarL·eiter und I ührer. Er hat nidit nursehr wertvolleErgehnisse seiner

Forsdierarbeit ihr zur Veröffentlichung anvertraut, er hat uns audi zum Vorteil unserer Leser mit Rat und Tat in den sdiweren Zeiten unserer jüngsten Vergangenheit zur Seite gestanden. $1 \mathrm{hm}$ dafür am heutigen Tage zu danken und zu huldigen, ist der Redaktion und dem Verlag ein herzlidies Bedürfnis!

Möge unserm Führer und Freund, möge dem großen Frauenarzt unserer Zeit nodi eine lange Reihe von Lustren beschiecíen sein, in denen er die Früdite seiner Arbeit aus-reifen sehen kann. Möge es ihm vergönnt sein, zu erleben, daß sein Werk, die moderne Frauenheilkunde, sich weiter in seinem Sinne entwickelt zu einer Periode unserer Ge-sdiichte, die den Namen „A I b e r t Döderlein" trägt!

Redaktion und Verlag der Monatsschrift für Geburtshilfe und Gynäkologie

A. Martin L. Seitz H. Sellheim G. A. Wagner S. Karger 